
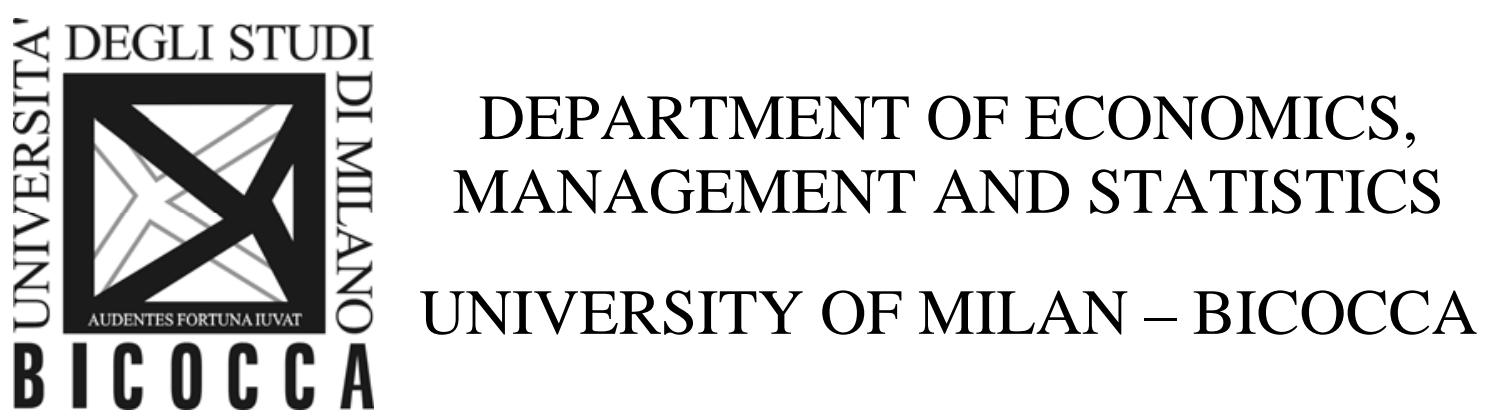

DEMS WORKING PAPER SERIES

\title{
Proportional Representation with Uncertainty
}

Francesco De Sinopoli, Giovanna Iannantuoni, Elena Manzoni, Carlos Pimienta

No. 288 - December 2014

Dipartimento di Economia, Metodi Quantitativi e Strategie di Impresa Università degli Studi di Milano - Bicocca

http://dems.unimib.it/ 


\title{
Proportional Representation with Uncertainty
}

\author{
Francesco De Sinopoli, Giovanna Iannantuoni ${ }^{\dagger}$ Elena Manzoni; \\ and Carlos Pimienta ${ }^{\S \mathbb{1}}$
}

December 22, 2014

\begin{abstract}
We introduce a model with strategic voting in a parliamentary election with proportional representation and uncertainty about voters' preferences. In any equilibrium of the model, most voters only vote for those parties whose positions are extreme. In the resulting parliament, a consensus government forms and the policy maximizing the sum of utilities of the members of the government is implemented.
\end{abstract}

JEL Classification Numbers: C72, D72.

Keywords: Proportional Election, Strategic Voting, Legislative Bargaining.

\footnotetext{
*Department of Economics, University of Verona. E-mail: francesco.desinopoli@univr.it

${ }^{\dagger}$ Corresponding author: Department of Economics, Management and Statistics, University of Milano-Bicocca. E-mail: giovanna.iannantuoni@unimib.it

${ }^{\ddagger}$ Department of Economics, Management and Statistics, University of Milano-Bicocca. Email: elena.manzoni@unimib.it

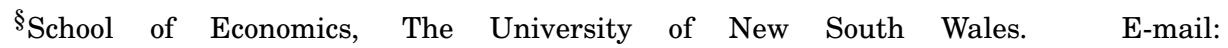
c.pimienta@unsw.edu.au

IWe received useful suggestions from Michela Cella, Leo Ferraris and Claudia Meroni. This paper was completed while Carlos was visiting University of Verona, their hospitality is gratefully acknowledged. Francesco, Giovanna and Elena thank financial support from PRIN 2010-2011 "New Approaches to political economy: positive political theories, empirical evidence and experiments in laboratory". Carlos thanks financial support from the Australian Research Council's Discovery Projects funding scheme DP140102426.
} 


\section{Introduction}

A central issue in the political economy literature is understanding how voters influence the policy outcome in a democracy. Voters select their political representatives through the electoral rule and politicians, in turn, choose the policy outcome. When elections are held under plurality rule, the system implements the policy preferred by the winner. As rational voters anticipate this, the theoretical and empirical literature has typically assumed that voters are instrumentally motivated in such a context (Cox, 1997). In the proportional representation case, however, voting incentives are more complicated. This seems to be the main reason behind the dearth of models that assume strategic voting under proportional representation. However, Cox (1997) also identifies the strategic incentives that voters face under proportional representation systems. More recently, Abramson et al. (2010) and Hobolt and Karp (2010) show that the amount of strategic voting under plurality and proportional system are quite similar.

But there is an additional layer of complexity. Typical modern democracies are characterized by a legislative body, elected by proportional rule, and by an executive body, which derives its mandate from the legislature. The theoretical literature on legislative bargaining under proportional representation starts from Austen-Smith and Banks (1988) where a quite complex three-party model is analyzed leading to a plethora of equilibria. The existence of a multiplicity of government structures that arise in equilibrium is a common characteristic of these models. This can be seen also in Baron and Diermeier (2001) who put forward a protocol of legislative bargaining in a three-party system and show how the government coalition and the policy outcome may depend on who is selected as government formateur.

Thus, policy oriented voters have to anticipate how their vote affects the final policy outcome in such a complex institutional setup. There seems to be evidence that this is indeed the case. Bargsted and Kedar (2009) show empirically that the expectation about which parties will form the government coalition influences voters' behavior. Duch et al. (2010) illustrate, using data of over 23 countries, that voters anticipate post-electoral bargaining outcomes and factor this expectation into their vote choice. Moreover, Kedar (2005) studies and tests empirically a model in which voters anticipate policy choices in parliamentary elections, hence displaying strategic behavior in this context. 
We introduce a tractable proportional representation model that incorporates all of these main features. Voters vote taking into account which government will eventually form and which will be the policy implemented by such a government. For the voting stage, we build on De Sinopoli and Iannantuoni (2007). The authors study strategic voting under proportional rule and find that, essentially, only a two-party equilibrium exists, in which rational voters vote only for the two extreme parties. However, we departure from their model by allowing for uncertainty over the voters' preferences, thus, enriching the model in a more realistic manner. For the government formation process and final policy choice, we draw on Baron and Diermeier (2001) who study a three party proportional model with a post-electoral bargaining stage. A randomly selected formateur chooses a potential government coalition and makes its members a take-it-or-leave-it offer over both the policy outcome and the allocation of transfers. In equilibrium, the policy choice corresponds to the policy that maximizes the sum of utilities of the government members. The main difference in this stage of our model with Baron and Diermeier (2001) is that legislators' preferences allow us to work with an arbitrary number of parties.

We obtain the following results. Most strategic voters vote only for two extreme parties at either side of the political spectrum. This is the most efficient manner in which a voter moves the final policy outcome towards her preferred policy as she anticipate that the final policy will be the average of the elected legislators' ideal points. Thus, the composition of the parliament is dominated by members of these two extreme parties, nonetheless, any selected formateur selects a government coalition consisting of every member of the parliament, that is, a consensus government arises. The implemented policy is the one that maximizes the sum of utilities of the members of the government.

Cho (2014) tackles a research question very close to ours, that is, the theoretical analysis of the strategic behavior of policy oriented voters in proportional representation elections with a post-electoral bargaining stage. Contrary to our findings, proportional representation in his framework promotes the representation of small parties. In a Romer and Rosenthal (1978) bargaining protocol he analyzes robust equilibria in which a voter's choice must remain best response when there is a small probability that the proposer selection is proportional even when there is a majority party (the Romer and 
Rosenthal (1978) protocol prescribes that a majority party is the proposer with probability one). Under such equilibria, voters have an incentive to vote for minority parties.

Studying strategic voting under proportional representation in our setting might shed light in various directions. First it can help understand how strategic voters' preferences are aggregated into outcomes in parliamentary elections. This, in turn, has implications on how many and which parties arise in this context. Our results suggest that strategic behavior under proportional representation leads to the polarization of parties' positions because extreme parties take most votes. The empirical political economy literature debates such a result since the seminal work of Cox (1997). Recently Kedar (2005) studies a decision theoretical voting model and shows empirically that proportional representation elections favors voting for extreme parties. Interestingly, the mechanisms at work is quite similar to ours: "voters compensate for the watering-down of their vote, often voting instrumentally for a party whose positions differ from their own".

The paper is organized as follows. Section 2 presents the model. We solve the post-electoral bargaining in Section 3 , the government coalition selection in Section 4, and the electoral stage in Section 5. Section 6 concludes the paper analyzing three examples.

\section{The Model}

We consider a political environment with policy space $\mathbb{X}=[0,1]$ and status quo $q \in \mathbb{X}$.

The set of parties is $P \equiv\{1, \ldots, k, \ldots, p\}$. Each party $k \in P$ has a commonly known ideal policy $\zeta_{k} \in[0,1]$. The representation that each party has in the parliament is decided by a finite set of voters $N=\{1, \ldots, i, \ldots, n\}$. Each voter $i \in N$ has private information about her ideal policy $\theta_{i} \in[0,1]$. Voters' ideal policies are independently distributed according to some commonly known distribution $F(\cdot)$ with support [0,1]. We assume that voter $i$ 's preferences can be represented by the quadratic utility function

$$
u_{i}\left(x, \theta_{i}\right)=-\left(x-\theta_{i}\right)^{2} .
$$

Voters select the composition of the parliament through a proportional electoral system. Hence, we let $S_{i}=\{\mathbf{1}, \ldots, \mathbf{k}, \ldots, \mathbf{p}\}$ be the set of ballots available to voter $i$. Ballot $s_{i}=\mathbf{k}$ is a vector of $p$ components with all zeros except 
for a one in the $k$-th position representing the vote for party $k$. Given a ballot profile $s=\left(s_{1}, \ldots, s_{n}\right)$, the share of votes accrued by party $k$ is equal to $\mu_{k}^{s} \equiv \frac{1}{n} \sum_{i=1}^{n} s_{i}^{k}$. Correspondingly, if the ballot profile is $s$, the share of seats that party $k$ has in the parliament is also equal to $\mu_{k}^{s}$. Thus, a ballot profile $s$ induces a composition of the parliament or a set of legislators $M^{s} \equiv\left\{1, \ldots, l, \ldots, m^{s}\right\}$. To abstract from issues related to the indivisibility of seats in the parliament we assume that, for every $s$, the size of the parliament $m^{s}$ is such that, for every party $k$, the expression $m^{s} \cdot \mu_{k}^{s}$ is an integer value. (This is satisfied if, e.g., $m^{s}=n$ for every ballot profile $s$.)

Each legislator $l \in M^{s}$ is characterized by her ideal policy $\zeta_{l} \in[0,1]$. Legislators have the same ideal policy as the party that they represent, therefore, for each party $k$, there are $m^{s} \cdot \mu_{k}^{s}$ legislators in $M^{s}$ whose ideal policy is equal to $\zeta_{k}$. Legislator $l$ 's preferences over policies are also represented by a quadratic utility function $u_{l}\left(x, \zeta_{l}\right)$ (as in, for example, Baron and Diermeier, 2001):

$$
u_{l}\left(x, \zeta_{l}\right)=-\left(x-\zeta_{l}\right)^{2} .
$$

A legislator may or may not belong to the government. If a legislator is not part of the government she only cares about the implemented policy and, therefore, her final utility is represented by (1). If a legislator belongs to the government then she also cares about office-holding benefits $B \in \mathbb{R}$ and transfers $y_{l} \in \mathbb{R}$. We assume that both office-holding benefits and transfers enter linearly in the utility function of the legislator. Therefore, if policy $x \in[0,1]$ is implemented, the utility to a legislator $l$ that belongs to the government and receives transfers $y_{l} \in \mathbb{R}$ is equal to

$$
U_{l}\left(x, y_{l}, \zeta_{l}\right)=B+u_{l}\left(x, \zeta_{l}\right)+y_{l}
$$

We furthermore assume that legislators are sufficiently office motivated. In particular, if we eliminate transfers, every legislator should prefer belonging to the government to the implementation of the status quo $q \in[0,1]$. Given preferences over policies in (1), this is guaranteed whenever $B>1 .^{1}$ Addi-

\footnotetext{
${ }^{1}$ Of course, we may have alternatively assumed that the utility to the legislators from the status quo is sufficiently low. This seems like weak hypothesis if the status quo is given by a new election. In this case legislators face the possibility of not being reelected and the cost of staying out of the new parliament, as in Austen-Smith and Banks (1988). See Gerber and Ortuño-Ortín (1998) for a similar assumption about a sufficiently disliked outcome in case of disagreement.
} 
tionally, transfers have to satisfy budget balance, so that $\sum_{l=1}^{m^{s}} y_{l} \leq 0$. (Note that $y_{l}=0$ whenever legislator $l$ is not in the government.)

The model is formalized as a three-stage game. In the first stage voters privately observe their type and cast their vote. Thus, a strategy for voter $i$ is a function $\sigma_{i}:[0,1] \rightarrow \Delta\left(S_{i}\right)$ from the set of possible types of voter $i$ to the set of probability distributions on ballots. The vector $\sigma_{i}\left(\theta_{i}\right)=\left(\sigma_{i}^{1}\left(\theta_{i}\right), \ldots, \sigma_{i}^{k}\left(\theta_{i}\right), \ldots, \sigma_{i}^{p}\left(\theta_{i}\right)\right)$ contains the probability that voter $i$ votes for each party when her ideal policy is $\theta_{i}$.

As mentioned above, the structure of the parliament is decided under proportional rule so, given a ballot profile $s$, each party $k$ 's proportion of seats in the parliament $M^{s}=\left\{1, \ldots, m^{s}\right\}$ is equal to its vote share $\mu_{k}^{s}$. In the second stage both the composition of the government and the policy are decided. We solve this stage by relying on the bargaining protocol proposed in Baron and Diermeier (2001). Every legislator $l \in M^{s}$ has the same probability to be selected as the formateur (we think of her as the selected prime minister). Under this bargaining protocol, the formateur, to be denoted as $f$, selects a coalition $G \subset M^{s}$ such that $f \in G$ and makes a take-it-or-leave-it offer both in policy and transfers dimensions. In case the coalition does not have a majority of seats in the parliament or at least one of the members of the coalition does not accept the take-it-or-leave-it offer then the government does not form.

In the third stage policy and transfers are implemented. If the formation of government $G$ was successful then policy $x^{G}$ is implemented and transfers are made according to the vector $y^{G}$. If the government formation process was unsuccessful then the status quo $q$ is implemented and no transfers are made. We now solve the game backward, starting from the analysis of the selection of policy outcome and transfers.

\section{Selection of policy outcome and transfers}

The third stage of the model consist of the implementation of the policy outcome and transfers determined by the second stage. In the second stage, a formateur is randomly selected among the legislators. As mentioned above, we use the bargaining protocol introduced in Baron and Diermeier (2001) to model and solve this stage.

Nature selects the formateur. The probability that each party has one of 
its member selected as the formateur is equal to its vote share. The formateur selects the government $G$, the policy $x^{G}$, and a vector of transfers $y^{G}$. We compute what the optimal policy and transfer vector are that maximize the formateur's utility for a given coalition $G$. That is,

$$
\begin{aligned}
\max _{x, y} U_{f}\left(x, y_{f}, \zeta_{f}\right) & \equiv B+u_{f}\left(x, \zeta_{f}\right)+y_{f} \\
\text { s.t. } & \\
B+u_{l}\left(x, \zeta_{l}\right)+y_{l} & \geq u_{l}\left(q, \zeta_{l}\right) \text { for all } l \in G \backslash\{f\}, \text { and } \\
0 & \geq \sum_{l \in G} y_{l} .
\end{aligned}
$$

Inequalities in (3) are the participation constraints for legislators in $G \backslash\{f\}$ and (4) is the budget balance constraint. Since the utility of the formateur is increasing in $y_{f}$, it is easy to show that the inequalities in (3) and (4) must be binding. Combine the resulting equalities in (3) and (4) to solve for $y_{f}$ and substitute the resulting expression in (2). We find that the optimal policy $x^{G}$ must be the utilitarian solution:

$$
x^{G} \in \arg \max _{x} \sum_{l \in G} u_{l}\left(x, \zeta_{l}\right),
$$

that is,

$$
x^{G}=\frac{1}{|G|} \sum_{l \in G} \zeta_{l} .
$$

Thus, the vector of transfers $y^{G}$ is given by:

$$
\begin{aligned}
y_{l}^{G} & =u_{l}\left(q, \zeta_{l}\right)-B-u_{l}\left(x^{G}, \zeta_{l}\right) & & \text { if } l \in G \backslash\{f\}, \\
y_{l}^{G} & =0 & & \text { if } l \notin G, \text { and } \\
y_{f}^{G} & =|G| B+\sum_{l \in G \backslash\{f\}}\left[u_{l}\left(x^{G}, \zeta_{l}\right)-u_{l}\left(q, \zeta_{l}\right)\right] . & &
\end{aligned}
$$

Under this policy choice and transfers, the utility of each member of the coalition $l \in G \backslash\{f\}$ other than the formateur is

$$
U_{l}\left(x^{G}, y_{l}^{G}, \zeta_{l}\right)=B+u_{l}\left(x^{G}, \zeta_{l}\right)+y_{l}^{G}=u_{l}\left(q, \zeta_{l}\right) .
$$

Whereas the utility to the formateur is

$$
U_{f}\left(x^{G}, y_{f}^{G}, \zeta_{f}\right)=B+u_{f}\left(x^{G}, \zeta_{f}\right)+y_{f}^{G}=|G| B+\sum_{l \in G} u_{l}\left(x^{G}, \zeta_{l}\right)-\sum_{l \in G \backslash\{f\}} u_{l}\left(q, \zeta_{l}\right) .
$$

We see that the bargaining power is in the formateur's hands who extracts all the rent associated to $x^{G}$. As a consequence, $U_{f}\left(x^{G}, y_{f}^{G}, \zeta_{f}\right) \geq U_{f}\left(q, 0, \zeta_{f}\right)$. 


\section{The Government Coalition Selection}

We now focus on the structure of the optimal coalition. We prove that the optimal size of the coalition always leads to consensus governments, that is governments that include every member of the parliament.

Proposition 1 Given that for each possible coalition $G$ the formateur proposes the policy $x^{G}$ and transfers $y^{G}$, in equilibrium, the government is composed of every legislator in the parliament.

Proof. Suppose that there is a legislator $j \notin G$, we prove that

$$
U_{f}\left(x^{G \cup\{j\}}, y_{f}^{G \cup\{j\}}, \zeta_{f}\right)>U_{f}\left(x^{G}, y_{f}^{G}, \zeta_{f}\right) .
$$

Using the utility to the formateur computed in the previous section, we rewrite this expression as

$$
\sum_{l \in G \cup\{j\}} u_{l}\left(x^{G \cup\{j\}}, \zeta_{l}\right)-\sum_{l \in G} u_{l}\left(x^{G}, \zeta_{l}\right)>u_{j}\left(q, \zeta_{j}\right)-B .
$$

Given that $x^{G \cup\{j\}}$ is the policy outcome that maximizes the sum of utilities of legislators in $G \cup\{j\}$, the left hand side of (6) satisfies

$$
\begin{aligned}
\sum_{l \in G \cup\{j\}} u_{l}\left(x^{G \cup\{j\}}, \zeta_{l}\right)-\sum_{l \in G} u_{l}\left(x^{G}, \zeta_{l}\right) \geq & \\
& \sum_{l \in G \cup\{j\}} u_{l}\left(x^{G}, \zeta_{l}\right)-\sum_{l \in G} u_{l}\left(x^{G}, \zeta_{l}\right)=u_{j}\left(x^{G}, \zeta_{j}\right) \geq-1,
\end{aligned}
$$

where the last inequality follows from the shape of the utility function. On the other hand, the right hand side of (6) satisfies

$$
u_{j}\left(q, \zeta_{j}\right)-B \leq-B<-1,
$$

establishing the desired result.

The proof of the previous proposition makes evident that the assumption that legislators are sufficiently office motivated, that is $B>1$, can be dispensed with if we assume that, for every legislator $j$, the status quo is at least as bad an outcome for her as her least preferred policy. ${ }^{2}$

\footnotetext{
${ }^{2}$ If we relax the assumption $B>1$ and we do not impose extra assumptions on the utility that the legislators derive from the status quo then the government coalition and the policy outcome would typically depend on the identity of the selected formateur (see Baron and Diermeier, 2001). In these cases, the model becomes intractable due to the wild multiplicity of equilibria caused by the typical coordination problem of the voting stage.
} 
We also note that the legislators may use any other bargaining protocol different from the one in Baron and Diermeier (2001) without modifying the results in the model as long as such a protocol yields the utilitarian solution.

\section{Strategic Voting}

Recall that a ballot profile $s=\left(s_{1}, \ldots, s_{n}\right)$ induces the vector of vote shares $\mu^{s} \equiv n^{-1} \sum_{i=1}^{n} s_{i}$. A type profile $\theta$, together with a strategy profile $\sigma$, induces a probability distribution over ballot profiles that results in the expected vector of vote shares $\mu^{\sigma(\theta)} \equiv n^{-1} \sum_{i=1}^{n} \sigma_{i}\left(\theta_{i}\right)$. We also define the vector of vote shares that is obtained if we remove player $i$, that is, $\mu^{\sigma_{-i}\left(\theta_{-i}\right)} \equiv(n-1)^{-1} \sum_{i=1}^{n} \sigma_{i}\left(\theta_{i}\right)$.

Given a strategy profile $\sigma$ we compute the expected vector of vote shares $\mu^{\sigma}$ by taking the expectation over type profiles $\mu^{\sigma} \equiv \mathbb{E}_{\theta}\left[\mu^{\sigma(\theta)}\right]$. Analogously, we also define $\mu^{\sigma_{-i}} \equiv \mathbb{E}_{\theta_{-i}}\left[\mu^{\sigma_{-i}\left(\theta_{-i}\right)}\right]$. It is also convenient to define player $i$ 's expected voting behavior $\mu^{\sigma_{i}} \equiv \mathbb{E}_{\theta_{i}}\left[\sigma_{i}\left(\theta_{i}\right)\right]$.

Given a vector of vote shares $\mu \in \Delta(P)$, the outcome that would result form the bargaining stage analyzed in the previous section is

$$
X(\mu)=\sum_{k=1}^{p} \zeta_{k} \mu_{k}
$$

The next proposition states that, apart from a small fraction of the electorate, policy-oriented voters vote only for the two most extreme parties whose bliss points are at either side of the policy space. Furthermore, the complement of this set of voters becomes negligible as size of the electorate $n$ grows to infinity. Let $L$ be the leftmost party and let $R$ be the rightmost party, that is, $L \equiv \arg \min _{k \in P} \zeta_{k}$, and $R \equiv \arg \max _{k \in P} \zeta_{k}$.

Proposition 2 Let $\sigma$ the be a voting strategy profile used in an equilibrium of the model. For every voter $i$,

( $\alpha$ ) if $\theta_{i} \leq X\left(\mu^{\sigma}\right)-\frac{1}{n}$ then $\sigma_{i}\left(\theta_{i}\right)=\boldsymbol{L}$, and

( $\beta$ ) if $\theta_{i} \geq X\left(\mu^{\sigma}\right)+\frac{1}{n}$ then $\sigma_{i}\left(\theta_{i}\right)=\boldsymbol{R}$.

Proof. With abuse of notation, for every party $k$, we let $\mathbf{k}$ denote both the pure action of some player $i$ that votes for party $k$ and the constant strategy that prescribes voting for party $k$ for every realization of $\theta_{i}$.

$(\alpha)$ We show that if $\theta_{i} \leq X\left(\mu^{\sigma}\right)-\frac{1}{n}$ then voter $i$ 's unique best reply is to vote for party $L$, that is

$$
\mathbb{E}_{\theta_{-i}}\left[\mathbb{E}_{s_{-i}}\left[u_{i}\left(X\left(\mu^{s_{-i}, \mathbf{L}}\right), \theta_{i}\right)\right]-\mathbb{E}_{s_{-i}}\left[u_{i}\left(X\left(\mu^{s_{-i}, \mathbf{k}}\right), \theta_{i}\right)\right]\right]>0 \quad \text { for all } \quad \mathbf{k} \in S_{i} \backslash\{\mathbf{L}\} .
$$


Using the quadratic form of the utility function we rewrite the previous inequality as:

$$
\mathbb{E}_{\theta_{-i}}\left[-\mathbb{E}_{s_{-i}}\left[\left(X\left(\mu^{s_{-i}, \mathbf{L}}\right)-\theta_{i}\right)^{2}\right]+\mathbb{E}_{s_{-i}}\left[\left(X\left(\mu^{s_{-i}, \mathbf{k}}\right)-\theta_{i}\right)^{2}\right]\right]>0 .
$$

Let $\operatorname{VAR}\left[X\left(\mu^{\sigma_{-i}\left(\theta_{-i}\right), \mathbf{k}}\right)\right]$ be the variance of $X\left(\mu^{\sigma_{-i}\left(\theta_{-i}\right), \mathbf{k}}\right)$. Expanding the previous expression, taking expectation with respect to other players' behavior, and using the properties of the variance of a random variable, we obtain

$$
\mathbb{E}_{\theta_{-i}}\left[\begin{array}{c}
-\left(X\left(\mu^{\sigma_{-i}\left(\theta_{-i}\right), \mathbf{L}}\right)-\theta_{i}\right)^{2}-\operatorname{VAR}\left[X\left(\mu^{\sigma_{-i}\left(\theta_{-i}\right), \mathbf{L}}\right)\right]+ \\
+\left(X\left(\mu^{\sigma_{-i}\left(\theta_{-i}\right), \mathbf{k}}\right)-\theta_{i}\right)^{2}+\operatorname{VAR}\left[X\left(\mu^{\sigma_{-i}\left(\theta_{-i}\right), \mathbf{k}}\right)\right]
\end{array}\right]>0,
$$

which simplifies to

$$
\mathbb{E}_{\theta_{-i}}\left[-\left(X\left(\mu^{\sigma_{-i}\left(\theta_{-i}\right), \mathbf{L}}\right)-\theta_{i}\right)^{2}+\left(X\left(\mu^{\sigma_{-i}\left(\theta_{-i}\right), \mathbf{k}}\right)-\theta_{i}\right)^{2}\right]>0 .
$$

Expanding again the previous expression and taking the expectation with respect to the distribution of voters' preferences, we get

$$
-\left[X\left(\mu^{\sigma_{-i}, \mathbf{L}}\right)-\theta_{i}\right]^{2}-\operatorname{VAR}\left[X\left(\mu^{\sigma_{-i}, \mathbf{L}}\right)\right]+\left[X\left(\mu^{\sigma_{-i}, \mathbf{k}}\right)-\theta_{i}\right]^{2}+\operatorname{VAR}\left[X\left(\mu^{\sigma_{-i}, \mathbf{k}}\right)\right]>0,
$$

which, similarly as above, simplifies to

$$
-\left[X\left(\mu^{\sigma_{-i}, \mathbf{L}}\right)-\theta_{i}\right]^{2}+\left[X\left(\mu^{\sigma_{-i}, \mathbf{k}}\right)-\theta_{i}\right]^{2}>0 .
$$

Rewrite this inequality as

$$
\left[X\left(\mu^{\sigma_{-i}, \mathbf{k}}\right)-X\left(\mu^{\sigma_{-i}, \mathbf{L}}\right)\right]\left[X\left(\mu^{\sigma_{-i}, \mathbf{k}}\right)+X\left(\mu^{\sigma_{-i}, \mathbf{L}}\right)-2 \theta_{i}\right]>0 .
$$

The first part of the last inequality is positive, i.e. $X\left(\mu^{\sigma_{-i}, \mathbf{k}}\right)>X\left(\mu^{\sigma_{-i}, \mathbf{L}}\right)$, because $L$ is the leftmost party. In order to prove our statement it is enough to prove $X\left(\mu^{\sigma_{-i}, \mathbf{L}}\right) \geq \theta_{i}$.

$$
\begin{aligned}
X\left(\mu^{\sigma_{-i}, \mathbf{L}}\right) & =\frac{n-1}{n} \sum_{k=1}^{m} \mu_{k}^{\sigma_{-i}} \zeta_{k}+\frac{1}{n} \zeta_{L} \\
& =\frac{n-1}{n} \sum_{k=1}^{m} \mu_{k}^{\sigma_{-i}} \zeta_{k}+\frac{1}{n} \sum_{k=1}^{m} \mu_{k}^{\sigma_{i}} \zeta_{k}-\frac{1}{n} \sum_{k=1}^{m} \mu_{k}^{\sigma_{i}} \zeta_{k}+\frac{1}{n} \zeta_{L} \\
& =X\left(\mu^{\sigma}\right)-\frac{1}{n} \sum_{k=1}^{m} \mu_{k}^{\sigma_{i}} \zeta_{k}+\frac{1}{n} \zeta_{L} \\
& \geq X\left(\mu^{\sigma}\right)-\frac{1}{n}\left(\zeta_{R}-\zeta_{L}\right) \geq X\left(\mu^{\sigma}\right)-\frac{1}{n} \geq \theta_{i} .
\end{aligned}
$$

( $\beta$ ) A similar argument applies. 
We conclude this section showing that the equilibrium outcome of the model is basically unique. In order to do so, let $\bar{F}$ be the correspondence whose graph is the closure of the graph of the distribution function $F$. Furthermore, define the correspondence $G$ so that, for each $\theta \in[0,1]$, the set $G(\theta)$ is the convex hull of $\bar{F}(\theta)$. (Note that $G$ is the correspondence $x \mapsto\{F(x)\}$ when the distribution function $F$ does not have any mass point.) Let $\theta^{*} \in[0,1]$ be the unique value that satisfies $\theta^{*} \in \zeta_{L} G\left(\theta^{*}\right)+\zeta_{R}\left(1-G\left(\theta^{*}\right)\right)$.

Proposition 3 If $\sigma$ is a voting strategy profile used in an equilibrium of the model then $\theta^{*}-\frac{1}{n} \leq X\left(\mu^{\sigma}\right) \leq \theta^{*}+\frac{1}{n}$.

Proof. Aiming for a contradiction, suppose $X\left(\mu^{\sigma}\right)<\theta^{*}-\frac{1}{n}$. Proposition 2 shows that $\sigma_{i}\left(\theta_{i}\right)=\mathbf{R}$ whenever $\theta_{i}>X\left(\mu^{\sigma}\right)+\frac{1}{n}$ which, in turn, implies $\sigma_{i}\left(\theta_{i}\right)=$ $\mathbf{R}$ whenever $\theta_{i} \geq \theta^{*}$. But, given the definition of $\theta^{*}$, it follows that $X\left(\mu^{\sigma}\right) \geq \theta^{*}$ because, under $\sigma$, party $R$ receives an expected share of votes of at least $\left(1-F\left(\theta^{*}\right)\right)$. This provides the desired contradiction, hence $\theta^{*}-\frac{1}{n} \leq X\left(\mu^{\sigma}\right)$. An analogous argument proves $X\left(\mu^{\sigma}\right) \leq \theta^{*}+\frac{1}{n}$.

\section{Examples}

We now introduce three examples that illustrate the main results of the paper. Consider an election where $n$ voters have to elect a parliament. There are three parties $L, C$ and $R$. The policy space is $[0,1]$, and $\zeta_{L}=0, \zeta_{C}=\frac{1}{2}$ and $\zeta_{R}=\frac{3}{5}$ the parties' bliss points.

Example 1: Discrete distribution of voters' bliss points. Let there be $n=7$ voters whose bliss points are independently drawn from the following distribution:

$$
\operatorname{Pr}\left(\theta_{i}=0\right)=\operatorname{Pr}\left(\theta_{i}=0.5\right)=\operatorname{Pr}\left(\theta_{i}=1\right)=\frac{1}{3} .
$$

Solving the game backward we know that that a consensus government arises and that the implemented policy is the average of the bliss points of the members of the coalition.

A voter with type $\theta_{i}=0$ always votes for party $L$ because, for any given composition of the parliament, she prefers a parliament with a higher share of legislators from party $L$. Similarly, voters with type $\theta_{i}=1$ always vote for party $R$. (These facts also follow from Propositions 2 and 3. Note that, in this example, $\theta^{*}=2 / 5$.) 
The optimal behavior of a voter with type $\theta_{i}=0.5$ depends on the likelihood of other voters' types and on their strategies. We show that in this case it is optimal for a voter with type $\theta_{i}=0.5$ to vote for party $R$ for any possible strategy profile such that type 0 voters vote for $L$ and type 1 voters vote for $R$.

Consider a voter with $\theta_{i}=0.5$ and the strategy profile $\hat{\sigma}_{-i}$ where for every $j \neq i$ :

$$
\hat{\sigma}_{j}\left(\theta_{j}\right)= \begin{cases}\mathbf{L} & \text { if } \theta_{j}=0, \\ (1-c-r ; c ; r) & \text { if } \theta_{j}=0.5, \\ \mathbf{R} & \text { if } \theta_{j}=1 .\end{cases}
$$

where centrist voters vote for parties $L, C$, and $R$ with respective probabilities $1-c-r, c$, and $r$. An argument along the lines of the proof of Proposition 2 shows that to analyze the voters' optimal behavior we can focus on the expected implemented policy. The expected implemented policy $X\left(\mu^{\hat{\sigma}_{-i}, \mathbf{R}}\right)$ when voter $i$ responds to $\hat{\sigma}_{-i}$ voting for $R$ is

$$
X\left(\mu^{\hat{\sigma}_{-i}, \mathbf{R}}\right)=\left(\frac{1}{3}\right)^{6}\left\{\frac{3645}{35} c+\frac{4374}{35} r+\frac{1254}{7}\right\} .
$$

where $c+r \leq 1$. Clearly, $X\left(\mu^{\hat{\sigma}_{-i}, \mathbf{R}}\right)$ is maximum when $r=1$. Hence consider the strategy profile $\sigma_{-i}^{*}$ where for every $j \neq i$

$$
\sigma_{j}^{*}\left(\theta_{j}\right)= \begin{cases}\mathbf{L} & \text { if } \theta_{j}=0 \\ \mathbf{R} & \text { otherwise }\end{cases}
$$

The expected implemented policy $X\left(\mu^{\sigma_{-i}^{*}}, \mathbf{R}\right)$ is

$$
X\left(\mu_{-i}^{\sigma_{-i}^{*}, \mathbf{R}}\right)=\left(\frac{1}{3}\right)^{6} \frac{10644}{35}=\frac{10644}{25515}<\frac{1}{2} .
$$

Therefore, the equilibrium strategy is $\sigma^{*}(\theta)$ where for any $i=1, \ldots, n$,

$$
\sigma_{i}^{*}\left(\theta_{i}\right)= \begin{cases}\mathbf{L} & \text { if } \theta_{i}=0 \\ \mathbf{R} & \text { otherwise }\end{cases}
$$

Example 2: Continuous distribution of voters' bliss points. We now consider the more general case with $n$ voters. We maintain the assumption of three parties with the same bliss points as before. In contrast, now voters' bliss points are independently drawn from the uniform distribution over $[0,1]$. As before, in the parliamentary bargaining stage of the game, a consensus government arises and the implemented policy is the average bliss point of the members of the coalition. 
In this example, Proposition 3 implies that the expected policy as $n$ grows to infinity is the policy $\theta^{*}$ that solves:

$$
\theta^{*}=F\left(\theta^{*}\right) \zeta_{L}+\left(1-F\left(\theta^{*}\right)\right) \zeta_{R},
$$

which in this example simplifies to $\theta^{*}=\left(1-\theta^{*}\right)(3 / 5)$, that is, $\theta^{*}=\frac{3}{8}$.

We now explicitly compute voting behavior for any given value of $n$. We start the analysis of voters' behavior from voters with extreme types. When a voter has type $\theta_{i}=0$, he votes for party $L$ for sure. Similarly, voters with type $\theta_{i} \geq \frac{3}{5}$ always vote for party $R$. The optimal behavior of a voter with type $\theta_{i} \in\left(0, \frac{3}{5}\right)$ depends instead on the expected behavior of the rest of the voters. Given that preferences are single-peaked, we have that:

- if a voter with bliss point $\hat{\theta}$ prefers $L$ to $C(R$ to $C$ ) then he also prefers $L$ to $R$ ( $R$ to $L)$;

- if a voter with bliss point $\hat{\theta}$ prefers $L$ to $C$ ( $R$ to $C$ ) then every voter $i$ with bliss point $\theta_{i}<\hat{\theta}\left(\theta_{i}>\hat{\theta}\right)$ prefers $L$ to both $C$ and $R$ ( $R$ to both $C$ and $L$ ).

For a given strategy profile of the other players, given that the utility function is quadratic, a voter with bliss point $\theta_{i}$ prefers $L$ to $C$ (and $R$ ) if:

$$
\left|\theta_{i}-X\left(\mu^{\sigma_{-i}, \mathbf{L}}\right)\right|<\left|\theta_{i}-X\left(\mu^{\sigma_{-i}, \mathbf{C}}\right)\right| .
$$

Similarly, a voter with bliss point $\theta_{i}$ prefers $R$ to $C$ (and $L$ ) if

$$
\left|\theta_{i}-X\left(\mu^{\sigma_{-i}, \mathbf{R}}\right)\right|<\left|\theta_{i}-X\left(\mu^{\sigma_{-i}, \mathbf{C}}\right)\right| .
$$

Let $\underline{\theta}$ be the bliss point of the voter who is indifferent between $L$ and $C$. Similarly, let $\bar{\theta}$ be the bliss point of the voter who is indifferent between $C$ and $R$. The voter's optimal strategy is

$$
\sigma_{i}\left(\theta_{i}\right)= \begin{cases}\mathbf{L} & \text { if } \theta_{i} \leq \underline{\theta}, \\ \mathbf{C} & \text { if } \underline{\theta}<\theta_{i}<\bar{\theta}, \\ \mathbf{R} & \text { if } \bar{\theta} \leq \theta_{i} .\end{cases}
$$

We can therefore write the policy outcomes as follows:

$$
\begin{aligned}
X\left(\mu^{\sigma_{-i}, \mathbf{L}}\right) & =\frac{3}{5} \frac{n-1}{n}(1-\bar{\theta})+\frac{1}{2} \frac{n-1}{n}(\bar{\theta}-\underline{\theta}), \\
X\left(\mu^{\sigma_{-i}, \mathbf{C}}\right) & =\frac{3}{5} \frac{n-1}{n}(1-\bar{\theta})+\frac{1}{2} \frac{n-1}{n}(\bar{\theta}-\underline{\theta})+\frac{1}{2} \frac{1}{n}, \\
X\left(\mu^{\sigma_{-i}, \mathbf{R}}\right) & =\frac{3}{5} \frac{n-1}{n}(1-\bar{\theta})+\frac{1}{2} \frac{n-1}{n}(\bar{\theta}-\underline{\theta})+\frac{3}{5} \frac{1}{n} .
\end{aligned}
$$


Using the indifference conditions for voters with types $\underline{\theta}$ and $\bar{\theta}$ we set up the following system of equations in two unknowns

$$
\begin{aligned}
& \underline{\theta}=\frac{3}{5} \frac{n-1}{n}(1-\bar{\theta})+\frac{1}{2} \frac{n-1}{n}(\bar{\theta}-\underline{\theta})+\frac{1}{4} \frac{1}{n}, \\
& \bar{\theta}=\frac{3}{5} \frac{n-1}{n}(1-\bar{\theta})+\frac{1}{2} \frac{n-1}{n}(\bar{\theta}-\underline{\theta})+\frac{11}{20} \frac{1}{n} .
\end{aligned}
$$

Solving for $\underline{\theta}$ and $\bar{\theta}$ we obtain

$$
\underline{\theta}=\frac{60 n^{2}-38 n+3}{20 n(8 n-3)} \quad \text { and } \quad \bar{\theta}=\frac{12 n^{2}+2 n-3}{4 n(8 n-3)} .
$$

Note that as $n$ goes to infinity both thresholds converge to $\frac{3}{8}$.

Voters with intermediate bliss points $\theta_{i} \in(\underline{\theta}, \bar{\theta})$ vote for party $C$. Notice that the fraction of these voters that do not vote for extreme parties is $(\bar{\theta}-\underline{\theta})=$ $\frac{1}{n} \frac{3}{10}$. Such a fraction is small even in small elections. For example, it is $5 \%$ for $n=6$, and $1 \%$ for $n=30$. If one considers an election with $n=1000$, as the election of a small town council, the fraction of voters with moderate behavior is $0,03 \%$.

Example 3: A change in voters' preferences. We now consider the effect that a change in voters' preferences has on the voting behavior of the electorate in Example 2. Suppose that $t$ voters become partisan supporters of party $L$, while the remaining $n-t$ voters are strategic policy motivated voters whose bliss points are distributed according to the uniform distribution over $[0,1]$.

As before, voters with bliss point $\theta_{i}=0$ vote for party $L$ and voters with bliss point $\theta_{i} \in\left(\frac{3}{5}, 1\right)$ vote for party $R$. Let $\underline{\theta}^{\prime}$ be the bliss point of a voter who is indifferent between $L$ and $C$, and let $\bar{\theta}^{\prime}$ be the bliss point of a voter who is indifferent between $C$ and $R$. As in Example 2, any voter $i$ 's optimal strategy is

$$
\sigma_{i}\left(\theta_{i}\right)=\left\{\begin{array}{l}
\mathbf{L} \quad \text { if } \theta_{i} \leq \underline{\theta}^{\prime}, \\
\mathbf{C} \text { if } \underline{\theta}^{\prime}<\theta_{i}<\bar{\theta}^{\prime}, \\
\mathbf{R} \text { if } \bar{\theta}^{\prime} \leq \theta_{i} .
\end{array}\right.
$$

We can therefore rewrite the policy outcomes as follows, taking into account 
that $k$ voters vote for party $L$.

$$
\begin{aligned}
& X\left(\mu^{\sigma_{-i}, \mathbf{L}}\right)=\frac{3}{5} \frac{n-t-1}{n}\left(1-\bar{\theta}^{\prime}\right)+\frac{1}{2} \frac{n-t-1}{n}\left(\bar{\theta}^{\prime}-\underline{\theta}^{\prime}\right), \\
& X\left(\mu^{\sigma_{-i}, \mathbf{C}}\right)=\frac{3}{5} \frac{n-t-1}{n}\left(1-\bar{\theta}^{\prime}\right)+\frac{1}{2} \frac{n-t-1}{n}\left(\bar{\theta}^{\prime}-\underline{\theta}^{\prime}\right)+\frac{1}{2} \frac{1}{n}, \\
& X\left(\mu^{\sigma_{-i}, \mathbf{R}}\right)=\frac{3}{5} \frac{n-t-1}{n}\left(1-\bar{\theta}^{\prime}\right)+\frac{1}{2} \frac{n-1}{n}\left(\bar{\theta}^{\prime}-\underline{\theta}^{\prime}\right)+\frac{3}{5} \frac{1}{n} .
\end{aligned}
$$

Using the indifference conditions for voters with types $\underline{\theta}^{\prime}$ and $\bar{\theta}^{\prime}$ we set up again a system of equations in two unknowns

$$
\begin{aligned}
& \underline{\theta}^{\prime}=\frac{3}{5} \frac{n-t-1}{n}\left(1-\bar{\theta}^{\prime}\right)+\frac{1}{2} \frac{n-t-1}{n}\left(\bar{\theta}^{\prime}-\underline{\theta}^{\prime}\right)+\frac{1}{4} \frac{1}{n}, \\
& \bar{\theta}^{\prime}=\frac{3}{5} \frac{n-t-1}{n}\left(1-\bar{\theta}^{\prime}\right)+\frac{1}{2} \frac{n-t-1}{n}\left(\bar{\theta}^{\prime}-\underline{\theta}^{\prime}\right)+\frac{11}{20} \frac{1}{n} .
\end{aligned}
$$

The solution to this system is

$$
\begin{gathered}
\underline{\theta}^{\prime}=\frac{60 n^{2}-60 n t-38 n+3(t+1)}{20 n(8 n-3(t+1))}, \\
\bar{\theta}^{\prime}=\frac{12 n^{2}-12 n t+2 n-3(t+1)}{32 n^{2}-12 n(t+1)} .
\end{gathered}
$$

Both thresholds are decreasing in $t$, implying $\underline{\theta}^{\prime}<\underline{\theta}$ and $\bar{\theta}^{\prime}<\bar{\theta}$. Intuitively, the higher the fraction of partisan voters voting for $L$, the greater the incentives for a moderate voter to vote for $R$ to compensate for the presence of leftist voters. However, this effect changes both thresholds in a way that the fraction of voters who do not vote for extreme parties does not change. That is, $\left(\bar{\theta}^{\prime}-\underline{\theta}^{\prime}\right)=\frac{1}{n} \frac{3}{10}$. Moreover, both thresholds also converge to $\frac{3}{8}$ as the number of voter $n$ grows to infinity.

\section{References}

[1] Abramson, P.R., Aldrich, J.H., Blais, A., Diamond, M., Diskin, A., Indridason I.H., Lee, D.J. and Levine, R. (2010). Comparing Strategic Voting under FTPT and PR. Comparative Political Studies, 43(1), 61-90.

[2] Austen-Smith, D. and Banks, J. (1988). Elections, Coalitions, and Legislative Outcomes. American Political Science Review, 82, 405-422.

[3] Bargsted, M.A. and Kedar, O. (2009). Coalition-Targeted Duvergerian Voting: How Expectations Affect Voter Choice under Proportional Representation. American Political Science Review, 82(2), 405-422. 
[4] Baron, D.P. and Diermeier, D. (2001). Elections, Governments, and Parliaments in Proportional Representation Systems. Quarterly Journal of Economics, 116, 933-967.

[5] Cho, S. (2014). Voting Equilibria under Proportional Representation. American Political Science Review, 108, 281-296.

[6] Cox, G.W. (1997). Making Votes Count: Strategic Coordination in the World's Electoral Systems. Cambridge, UK: Cambridge University Press.

[7] De Sinopoli, F. and Iannantuoni, G. (2007). A Spatial Voting Model where Proportional Rule Leads to Two-Party Equilibria. International Journal of Game Theory, 35, 267-286.

[8] Duch, R.M., May, J. and Armstrong, D.A. (2010), Coalition-directed Voting in Multiparty Democracies. American Political Science Review, 104, 698-719.

[9] Gerber, A. and Ortuño-Ortín, I. (1998). Political Compromise and Endogenous Formation of Coalitions. Social Choice and Welfare, 15, 445454.

[10] Hobolt, S.B. and Karp, J.A. (2010). Voters and Coalition Governments. Electoral Studies, 29, 299-307.

[11] Kedar, O. (2005). When Moderate Voters Prefer Extreme Parties: Policy Balancing in Parliamentary Elections. American Political Science Review, 99, 185-199.

[12] Romer, T. and Rosenthal H. (1978). Political Resource Allocation, Controlled Agendas, and the Status Quo. Public Choice, 33, 27-43. 